\title{
Capacity of a Structural Binary Symmetric Channel
}

\author{
Lan V. Truong \\ School of Electrical and Computer Engineering \\ Purdue University, West Lafayette, IN, USA \\ Email: truongl@purdue.edu
}

\author{
Wojciech Szpankowski \\ Department of Computer Science \\ Purdue University, West Lafayette, IN, USA \\ Email: spa@cs.purdue.edu
}

\begin{abstract}
Information theory traditionally deals with the problem of transmitting sequences over a communication channel and find the maximum number of messages that transmitter can send so that the receiver recovers these messages with arbitrarily small probability of error. However, databases of various sorts have come into existence in recent years that require to transmit new sources of data (e.g., graphs and sets) over communication channels. In this paper, we investigate a communication model where we need to transmit an ErdôsRényi graph to a destination over a Binary Symmetric Channel (BSC). We find the capacity of such a channel, called Structural Binary Symmetric Channel (SBSC), to be $C=1-h(\epsilon)$ where $\epsilon$ is the error bit rate and $h(\epsilon)$ is the binary entropy.
\end{abstract}

\section{INTRODUCTION}

In 2003 Brooks [2] observed that there is no information theory that gives us a metric for information embodied in structure. Such investigations should be of particular interest to biology as recently opined by P. Nurse [8] (e.g., in biology information is often coded in structure, for example of a protein). Nurse argued that focusing on information flow will help to understand better how cells and organisms work. This opens unbounded opportunities for information theory to extend its scope beyond its original goals, that of communication and storage. We suggest [3], [10] to broaden information theory to study finite size data structures (e.g., graphs, sets, social networks), that is, to develop information theory of data structures beyond first-order asymptotics. In
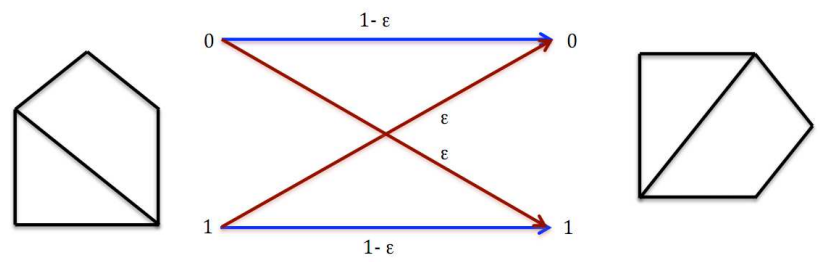

Fig. 1. Effect of BSC Channel on Graphs

particular, in [3] as the first step in understanding structural information, we explore structures on graphs, specifically, we study unlabeled graphs (or structures) and defined structural entropy characterizing graph compression.

In this paper, we move one step further and investigate how much structural information can be recovered when a structure (unlabeled graph) is transmitted over a noisy channel, as shown in Figure 1. Observe that - due to graph symmetry - an unlabeled graph transmitted over a noisy channel can have the same structure on the receiving side even if errors occur, as illustrated in the next example.

Example. Let us consider a graph on four vertices $G_{1}=$ $\{A, B, C, D\}$ presented on the left-side of Figure 2 . On the
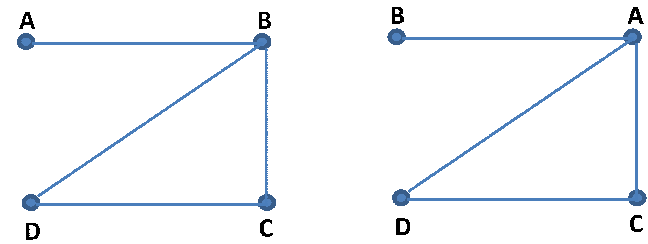

Fig. 2. Two identical unlabeled graphs

right-hand side we draw graph $G_{2}$ on the same set of vertices with labels $A$ and $B$ switched. Thus both graphs have the same structure (i.e., the same unlabeled graph). Observe that the adjacency matrices of these two graphs are quite different. In fact, we can obtain graph $G_{2}$ as an output of a binary symmetric channel with input $G_{1}$ and the following error matrix:

$$
Z=\left|\begin{array}{llll}
0 & 0 & 1 & 1 \\
0 & 0 & 1 & 1 \\
1 & 1 & 0 & 0 \\
1 & 1 & 0 & 0
\end{array}\right|
$$

Thus a natural question arises: how much "structural information" can be reliably transmitted over a noisy channel?

More precisely, here we analyze a communication system presented in Figure 3. Input messages consist of (unlabeled) Erdős-Rényi graphs $\mathcal{G}(n, p)$ (i.e., structures $S$ ) over $n$ vertices in which edges are added independently and randomly with probability $p$. These input graphs are encoded as adjacency matrices (sequences of length $\left(\begin{array}{l}n \\ 2\end{array}\right)$ ) by a graph encoder. Adjacency matrices are transmitted through a Binary Symmetric Channel (BSC) bit by bit. The graph decoder decodes the received matrices (sequences), then forms the estimation of the transmitted structure. We assume that the graph decoder knows the number of vertices of the transmitted graphs, hence the received graphs are random graphs over $n$ vertices. Observe that some edges of the transmitted graphs can be deleted or added, therefore the transmitted and received graphs may differ even if they unlabeled graphs - or structures - are indistinguishable. We shall see that the received graphs $\mathcal{G}^{\prime}$ are still Erdős-Rényi graphs. In short, in our setting an unlabeled transmitted graph is correctly decoded if the 


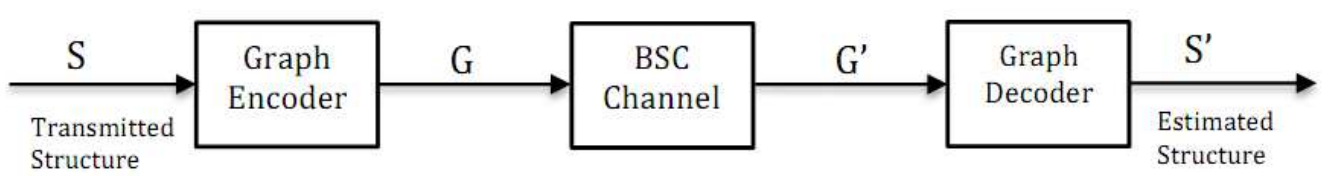

Fig. 3. Model of Graph Transmission over BSC Channel

transmitted and received graphs have the same structure (i.e., adjacency matrix). We call such a system the Structural Binary Symmetric Channel (SBSC), and study its capacity. The main result of this paper is presented next.

Theorem 1: Capacity of the structural Binary Symmetric Channel $\operatorname{SBSC}(\epsilon)$ is

$$
C=1-h(\epsilon)
$$

where $\epsilon$ is the error bit rate and $h(\epsilon)=-\epsilon \log \epsilon-(1-$ $\epsilon) \log (1-\epsilon)$ is the binary entropy.

The literature on structural compression (entropy) and transmission of structures over noisy channel is quite limited. In 1984, Turan [11] raised the question of finding an efficient coding method for general unlabeled graphs on $n$ vertices, suggesting a lower bound of $\left(\begin{array}{l}n \\ 2\end{array}\right)-n \log n+O(n)$ bits. In 1990 , Naor [7] proposed such a representation that is optimal up to the first two leading terms when all unlabeled graphs are equally likely. Naor's result is asymptotically a special case of ours when $p=1 / 2$. Finally, in a recent paper Kieffer et al. [6] presented a structural complexity of a binary tree. The structural entropy was introduced in [3] where the first provable (asymptotically) optimal graph compressor for Erdős-Rényi graph models was presented. To the best of our knowledge structural binary symmetric channels were not discussed in open literature.

\section{STRUCTURAL ENTROPY AND MUtUAL INFORMATION}

Given $n$ distinguishable vertices, a random graph is generated by adding edges randomly according to some distribution. Such a random graph model $\mathcal{G}$ produces a probability distribution on graphs, and the graph entropy $H(G)$ is defined naturally as

$$
H(G)=\mathbf{E}[-\log P(G)]=-\sum_{G \in \mathcal{G}} P(G) \log P(G)
$$

where $P(G)$ is the probability of a graph $G$. In [3], the authors introduced a random structure model $S$ for the unlabeled version of a random graph model $\mathcal{G}$. In such a model, graphs are generated in the same manner as in $\mathcal{G}$, but they are thought of as unlabeled graphs. That is, the vertices are indistinguishable, and the graphs having "the same structure" are considered to be the same even if their labeled versions are different. Thus, we shall use the terms unlabeled graphs and structures interchangeably.

The probability of $S$ can be computed as

$$
P(S)=\sum_{G \cong S, G \in \mathcal{G}} P(G)=M(S) P(G)
$$

where $G \cong S$ means that $G$ and $S$ have the same structure (i.e., they are isomorphic), and $M(S)$ denotes the number of labeled graphs having common structure $S$. It is well known that

$$
M(S)=\frac{n !}{|\operatorname{Aut}(S)|}
$$

where $\operatorname{Aut}(S)$ denotes automorphism of graph $G$ such that $G \cong S$ (so we also write $\operatorname{Aut}(G)$ ). We recall that an automorphism of a graph $G$ is an adjacency preserving permutation of the vertices of $G$. The structural entropy $H(S)$ of a random graph $G$ is then defined as [3]

$$
H(S)=\mathbf{E}[-\log P(S)]=-\sum_{S \in \mathcal{G}} P(S) \log P(S)
$$

where the summation is over all distinct structures.

In this paper, we introduce the structural mutual information. When one transmits a sequence of structures (unlabeled graphs) over a communication channel (e.g. SBSC channel), then the received sequence represents structures of some unlabeled graphs. The structural mutual information between the transmitted structure $S$ and received structure $S^{\prime}$ is then defined as

$$
I\left(S ; S^{\prime}\right)=\mathbf{E}\left[\log \frac{P\left(S, S^{\prime}\right)}{P(S) P\left(S^{\prime}\right)}\right] .
$$

In order to estimate the mutual information, and ultimately the capacity of the structural BSC, we need to specify the input. We shall assume that we transmit structures or unlabeled Erdős-Rényi graphs defined as follows. In the ErdősRényi random graph model $\mathcal{G}(n, p)$, graphs are generated randomly on $n$ vertices with edges chosen independently with probability $0<p<1$. If a graph $G$ in $\mathcal{G}(n, p)$ has $k$ edges, then $P(G)=p^{k}(1-p)^{\left(\begin{array}{l}n \\ 2\end{array}\right)-k}$. Let $\mathcal{S}(n, p)$ be the random structure model (unlabeled graphs) corresponding to $\mathcal{G}(n, p)$. Then, by (1) the probability of a structure $S$ (unlabeled version of $S$ becomes $P(S)=M(S) \cdot p^{k}(1-p)^{\left(\begin{array}{c}n \\ 2\end{array}\right)-k}$.

\section{Preliminary Results}

In this section we list some properties of the received structures over SBSC. In particular, we show that such a structure is still a random Erdôs-Rényi graph. We also estimate the structural entropy and the conditional entropy.

Lemma 1: Let $G \in \mathcal{G}(n, p)$ be transmitted over a $\operatorname{BSC}(\epsilon)$ channel and $G^{\prime}$ be the received graph. Then

(i) $G^{\prime} \in \mathcal{G}^{\prime}(n, p * \epsilon)$, where $p * \epsilon:=p(1-\epsilon)+\epsilon *(1-p)$.

(ii) $G^{\prime}=G \oplus Z$ where $Z \in \mathcal{G}(n, \epsilon)$, where $\oplus$ is the modulo-2 addition of the corresponding adjacency matrices. 
(iii) The conditional probability $P\left(G^{\prime} \mid G\right)=P(Z)$, where $Z=Z_{1} Z_{2} \ldots Z_{m}$ are $m=\left(\begin{array}{c}n \\ 2\end{array}\right)$ independent Bernoulli random variables with parameter $\epsilon$.

(iv) The conditional entropy is

$$
H\left(G^{\prime} \mid G\right)=\left(\begin{array}{l}
n \\
2
\end{array}\right) h(\epsilon) .
$$

Proof: We first prove (i). Assume $U$ is an edge of the transmitted graph $G \in \mathcal{G}(n, p)$ and $V$ is the corresponding edge of the received graph $G^{\prime}$. Then

$$
\begin{gathered}
P(V=1)=P(V=1 \mid U=0) P(U=0)+ \\
P(V=1 \mid U=1) P(U=1)=\epsilon(1-p)+(1-\epsilon) p:=p * \epsilon .
\end{gathered}
$$

Every edge of the received graph appears with the probability $p * \epsilon$, therefore the entire received graph is an Erdős-Rényi graph $\mathcal{G}^{\prime}(n, p * \epsilon)$.

For part (ii) we observe that since each graph $G \in \mathcal{G}(n, p)$ is composed of $m=\left(\begin{array}{l}n \\ 2\end{array}\right)$ edges, we need $m=\left(\begin{array}{l}n \\ 2\end{array}\right)$ transmissions to send the entire graph through the $\operatorname{BSC}(\epsilon)$ channel. Each edge is subject to noise resulting in adding Bernoulli random variables $Z_{i}$ to the corresponding edge (binary bit representing the edge in the adjacency matrix), where $P\left(Z_{i}=1\right)=\epsilon$ and $P\left(Z_{i}=0\right)=1-\epsilon$. This can be viewed as adding an Erdôs-Rényi (noise) graph $Z \in \mathcal{G}(n, \epsilon)$ to the transmitted $G \in \mathcal{G}(n, p)$ graph. Therefore, $G^{\prime}=G \oplus Z$ where $G \in \mathcal{G}(n, p), G^{\prime} \in \mathcal{G}(n, p * \epsilon)$, and $Z \in \mathcal{G}(n, \epsilon)$.

For (iii) observe that the transmitted graph instance $G \in$ $\mathcal{G}(n, p)$ can be considered as a binary sequence $\mathbf{X}=$ $\left(X_{1}, X_{2}, \ldots, X_{m}\right)$ of length $m=\left(\begin{array}{l}n \\ 2\end{array}\right)$. Similarly, the received graph instance $G^{\prime} \in \mathcal{G}^{\prime}(n, p * \epsilon)$ can be viewed as a binary sequence $\mathbf{Y}=\left(Y_{1}, Y_{2}, \ldots, Y_{m}\right)$. Therefore, we have:

$$
\begin{gathered}
P\left(G^{\prime} \mid G\right)=P\left(\mathbf{Y}_{1}^{m} \mid \mathbf{X}_{1}^{m}\right) \stackrel{(a)}{=} \prod_{k=1}^{m} P\left(Y_{k} \mid X_{k}\right) \\
\stackrel{(b)}{=} \prod_{k=1}^{m} P\left(Z=Z_{k}\right) \stackrel{(c)}{=} P\left(Z_{1} Z_{2} \ldots Z_{m}\right) .
\end{gathered}
$$

Here (a) follows form the memoryless property of the BSC channel, (b) follows from (ii) since $Y_{k}=X_{k} \oplus Z_{k}$; and (c) follows from properties of the BSC channel.

For part (iv), we notice that from (iii), we know that

$$
\begin{aligned}
H\left(G^{\prime} \mid G\right) & =E[-\log P(\mathbf{Z})]=E\left[-\log P\left(Z_{1} Z_{2} \ldots Z_{m}\right)\right] \\
& =\sum_{k=1}^{m} E\left[-\log P\left(Z_{k}\right)\right]=\sum_{k=1}^{m} H\left(Z_{k}\right)
\end{aligned}
$$

since $Z_{1}, Z_{2}, \ldots, Z_{m}$ are independent. On the other hand, since $Z_{k} \sim \operatorname{Bern}(\epsilon)$ for all $k=1,2, \ldots, m$, hence $H\left(Z_{k}\right)=$ $h(\epsilon)$, where $h(\epsilon)=-\epsilon \log \epsilon-(1-\epsilon) \log (1-\epsilon)$ is the entropy rate of memoryless binary source. Finally,

$$
H\left(G^{\prime} \mid G\right)=\left(\begin{array}{l}
n \\
2
\end{array}\right) h(\epsilon)
$$

for any fixed transmitted graph $G \in \mathcal{G}(n, p)$.

In the next lemma we present properties of the received structures over a noisy channel.
Lemma 2: Under the same assumptions as in Lemma 1: (i) Let $\left\{G_{1}, G_{2}, \ldots, G_{M(S)}\right\}$ be the set of all distinct labeled graphs of $\mathcal{G}(n, p)$ with structure $S$, and $\left\{G_{1}^{\prime}, G_{2}^{\prime}, \ldots, G_{M\left(S^{\prime}\right)}^{\prime}\right\}$ be the set of all labeled graphs of $\mathcal{G}^{\prime}(n, p * \epsilon)$ with structure $S^{\prime}$. Then for every $k$ the set

$$
\left\{G_{k} \oplus G_{1}^{\prime}, G_{k} \oplus G_{2}^{\prime}, \ldots, G_{k} \oplus G_{M\left(S^{\prime}\right)}^{\prime}\right\}
$$

for $k \in\{1,2, \ldots, M(S)\}$ a is vertex-permutation of

$$
\left\{G_{1} \oplus G_{1}^{\prime}, G_{1} \oplus G_{2}^{\prime}, \ldots, G_{1} \oplus G_{M\left(S^{\prime}\right)}^{\prime}\right\} .
$$

(ii) Furthermore,

$$
P\left(S^{\prime} \mid S\right)=\sum_{l=1}^{M\left(S^{\prime}\right)} P\left(G_{l}^{\prime} \mid G_{1}\right)
$$

where $G_{1}$ is a graph with structure $S$, and $\left\{G_{1}^{\prime}, G_{2}^{\prime}, \ldots, G_{M\left(S^{\prime}\right)}^{\prime}\right\}$ is the set of graphs with structure $S^{\prime}$.

(iii) The conditional entropy $H\left(S^{\prime} \mid S\right)$ satisfies

$$
H\left(S^{\prime} \mid S\right) \geq \log n !-\sum_{S^{\prime} \in \mathcal{G}^{\prime}} \log \left|\operatorname{Aut}\left(S^{\prime}\right)\right| P\left(S^{\prime}\right)+\left(\begin{array}{l}
n \\
2
\end{array}\right) h(\epsilon) .
$$

Proof: We start with part (i). For any $k \in$ $\{1,2, \ldots, M(S)\}$ since $G_{k}$ and $G_{1}$ have the same structure, there exists a vertex-permutation $\pi_{k}$ such that $\pi_{k}\left(G_{1}\right)=G_{k}$. Therefore, we have

$$
\begin{gathered}
\left\{G_{k} \oplus G_{1}^{\prime}, G_{k} \oplus G_{2}^{\prime}, \ldots, G_{k} \oplus G_{M\left(S^{\prime}\right)}^{\prime}\right\} \\
=\left\{\pi_{k}\left(G_{1}\right) \oplus G_{1}^{\prime}, \pi_{k}\left(G_{1}\right) \oplus G_{2}^{\prime}, \ldots, \pi_{k}\left(G_{1}\right) \oplus G_{M\left(S^{\prime}\right)}^{\prime}\right\} \\
=\left\{\pi_{k}\left(G_{1} \oplus \pi_{k}^{-1}\left(G_{1}^{\prime}\right)\right), \ldots, \pi_{k}\left(G_{1} \oplus \pi_{k}^{-1}\left(G_{M\left(S^{\prime}\right)}^{\prime}\right)\right\}\right.
\end{gathered}
$$

where $\pi^{-1}$ denotes the inverse vertex permutation of $\pi$. Observe that since $\left\{G_{1}^{\prime}, G_{2}^{\prime}, \ldots, G_{M\left(S^{\prime}\right)}^{\prime}\right\}$ is the set of all different labeled graphs of $\mathcal{G}^{\prime}(n, p * \epsilon)$ with structure $S^{\prime}$, therefore $\left\{\pi_{k}^{-1}\left(G_{1}^{\prime}\right), \pi_{k}^{-1}\left(G_{2}^{\prime}\right), \ldots, \pi_{k}^{-1}\left(G_{M\left(S^{\prime}\right)}^{\prime}\right)\right\}$ is the same set as $\left\{G_{1}^{\prime}, G_{2}^{\prime}, \ldots, G_{M\left(S^{\prime}\right)}^{\prime}\right\}$ although the later is a permutation of the former. This also means that

$$
\left\{\pi_{k}\left(G_{1} \oplus \pi_{k}^{-1}\left(G_{1}^{\prime}\right)\right), \ldots, \pi_{k}\left(G_{1} \oplus \pi_{k}^{-1}\left(G_{M\left(S^{\prime}\right)}^{\prime}\right)\right\}\right.
$$

is a permutation of

$$
\left.\pi_{k}\left(G_{1} \oplus G_{1}^{\prime}\right), \pi_{k}\left(G_{1} \oplus G_{2}^{\prime}\right), \ldots, \pi_{k}\left(G_{1} \oplus G_{M\left(S^{\prime}\right)}^{\prime}\right)\right\} .
$$

Therefore, $\left\{G_{k} \oplus G_{1}^{\prime}, G_{k} \oplus G_{2}^{\prime}, \ldots, G_{k} \oplus G_{M\left(S^{\prime}\right)}^{\prime}\right\}$ is a vertexpermutation of $\left\{G_{1} \oplus G_{1}^{\prime}, G_{1} \oplus G_{2}^{\prime}, \ldots, G_{1} \oplus G_{M\left(S^{\prime}\right)}^{\prime}\right\}$ for any $k \in\{1,2, \ldots, M(S)\}$.

Now we look at part (ii). Observe that

$$
\begin{aligned}
& P\left(S, S^{\prime}\right)=\sum_{k=1}^{M(S)} \sum_{l=1}^{M\left(S^{\prime}\right)} P\left(G_{k}, G_{l}^{\prime}\right) \\
& =\sum_{k=1}^{M(S)} P\left(G_{k}\right) \sum_{l=1}^{M\left(S^{\prime}\right)} P\left(G_{l}^{\prime} \mid G_{k}\right) .
\end{aligned}
$$

Since $G_{1}, G_{2}, \ldots, G_{M(S)}$ have the same structure $S$, $P\left(G_{1}\right)=P\left(G_{2}\right)=\ldots=P\left(G_{M(S)}\right)$. Therefore, we obtain

$$
P\left(S, S^{\prime}\right)=P\left(G_{1}\right) \sum_{k=1}^{M(S)} \sum_{l=1}^{M\left(S^{\prime}\right)} P\left(G_{l}^{\prime} \mid G_{k}\right)
$$


On the other hand, from part (i), we know that $\left\{G_{k} \oplus\right.$ $\left.G_{1}^{\prime}, G_{k} \oplus G_{2}^{\prime}, \ldots, G_{k} \oplus G_{M\left(S^{\prime}\right)}^{\prime}\right\}$ are vertex-permutations of $\left\{G_{1} \oplus G_{1}^{\prime}, G_{1} \oplus G_{2}^{\prime}, \ldots, G_{1} \oplus G_{M\left(S^{\prime}\right)}^{\prime}\right\}$ for any $k \in$ $\{1,2, \ldots, M(S)\}$. Therefore, for any $k \in\{1,2, \ldots, M(S)\}$ we must have for all $1 \leq i \leq M\left(S^{\prime}\right)$

$$
P\left(G_{i}^{\prime} \mid G_{k}\right)=P\left(Z=G_{k} \oplus G_{i}^{\prime}\right)=
$$

$\left.\stackrel{(a)}{=} P\left(Z=\pi_{k}\left(G_{1} \oplus G_{i}^{\prime}\right)\right) \stackrel{(b)}{=} P\left(Z=G_{1} \oplus G_{i}^{\prime}\right)\right)=P\left(G_{i}^{\prime} \mid G_{1}\right)$

where $Z \in \mathcal{G}^{\prime}(n, p * \epsilon)$. Here, (a) is a direct consequence of (i), and (b) follows from the fact that any pair of graph instances of the Erdős-Rényi noise graph $\mathcal{G}(n, \epsilon)$, say $G_{1}+G_{l}^{\prime}$ and $\pi_{k}\left(G_{1}+G_{l}^{\prime}\right)$ for any $l \in\left\{1,2, \ldots, M\left(S^{\prime}\right)\right\}$ (which are vertex-permutations of each other) have the same probability. This means that for any $k \in\{1,2, \ldots, M(S)\}$, we have

$$
\sum_{l=1}^{M\left(S^{\prime}\right)} P\left(G_{l}^{\prime} \mid G_{k}\right)=\sum_{l=1}^{M\left(S^{\prime}\right)} P\left(G_{l}^{\prime} \mid G_{1}\right) .
$$

From (2) and (3), we obtain

$$
P\left(S, S^{\prime}\right)=P(S) \sum_{l=1}^{M\left(S^{\prime}\right)} P\left(G_{l}^{\prime} \mid G_{1}\right)
$$

since $P(S)=P\left(G_{1}\right) M(S)$. Hence,

$$
P\left(S^{\prime} \mid S\right)=\sum_{l=1}^{M\left(S^{\prime}\right)} P\left(G_{l}^{\prime} \mid G_{1}\right) .
$$

For part (iii) we need to establish a bound on the conditional entropy $H\left(S^{\prime} \mid S\right)$. For this we first consider the convex function $f(x)=x \log x$ for $x>0$. Applying Jensen's inequality, we have

$$
f\left(\frac{\sum_{k=1}^{M\left(S^{\prime}\right)} P\left(G_{k}^{\prime} \mid G_{1}\right)}{M\left(S^{\prime}\right)}\right) \leq \frac{1}{M\left(S^{\prime}\right)} \sum_{k=1}^{M\left(S^{\prime}\right)} f\left(P\left(G_{k}^{\prime} \mid G_{1}\right) .\right.
$$

Hence, by (4)

$$
f\left(\frac{P\left(S^{\prime} \mid S\right)}{M\left(S^{\prime}\right)}\right) \leq \frac{1}{M\left(S^{\prime}\right)} \sum_{k=1}^{M\left(S^{\prime}\right)} f\left(P\left(G_{k}^{\prime} \mid G_{1}\right) .\right.
$$

Thus

$$
\begin{gathered}
\frac{P\left(S^{\prime} \mid S\right)}{M\left(S^{\prime}\right)} \log \left(\frac{P\left(S^{\prime} \mid S\right)}{M\left(S^{\prime}\right)}\right) \\
\leq \frac{1}{M\left(S^{\prime}\right)} \sum_{k=1}^{M\left(S^{\prime}\right)} P\left(G_{k}^{\prime} \mid G_{1}\right) \log P\left(G_{k}^{\prime} \mid G_{1}\right)
\end{gathered}
$$

or

$$
P\left(S^{\prime} \mid S\right) \log \left(\frac{P\left(S^{\prime} \mid S\right)}{M\left(S^{\prime}\right)}\right) \leq \sum_{k=1}^{M\left(S^{\prime}\right)} P\left(G_{k}^{\prime} \mid G_{1}\right) \log P\left(G_{k}^{\prime} \mid G_{1}\right) .
$$

Therefore,

$$
\begin{aligned}
& -\sum_{S^{\prime} \in \mathcal{G}^{\prime}} \sum_{k=1}^{M\left(S^{\prime}\right)} P\left(G_{k}^{\prime} \mid G_{1}\right) \log P\left(G_{k}^{\prime} \mid G_{1}\right) \\
& \leq-\sum_{S^{\prime} \in \mathcal{G}^{\prime}} P\left(S^{\prime} \mid S\right) \log \left(\frac{P\left(S^{\prime} \mid S\right)}{M\left(S^{\prime}\right)}\right) .
\end{aligned}
$$

By Lemma 2 (iii)

$$
\begin{gathered}
-\sum_{S^{\prime} \in \mathcal{G}^{\prime}} \sum_{k=1}^{M\left(S^{\prime}\right)} P\left(G_{k}^{\prime} \mid G_{1}\right) \log P\left(G_{k}^{\prime} \mid G_{1}\right) \\
=\sum_{G^{\prime} \in \mathcal{G}^{\prime}}-P\left(G^{\prime} \mid G_{1}\right) \log P\left(G^{\prime} \mid G_{1}\right)=H\left(G^{\prime} \mid G_{1}\right)=\left(\begin{array}{c}
n \\
2
\end{array}\right) h(\epsilon) .
\end{gathered}
$$

Therefore, from (5), we obtain

$$
\begin{gathered}
\left(\begin{array}{l}
n \\
2
\end{array}\right) h(\epsilon) \leq-\sum_{S^{\prime} \in \mathcal{G}^{\prime}} P\left(S^{\prime} \mid S\right) \log \left(\frac{P\left(S^{\prime} \mid S\right)}{M\left(S^{\prime}\right)}\right) \\
=-\sum_{S^{\prime} \in \mathcal{G}^{\prime}} P\left(S^{\prime} \mid S\right) \log P\left(S^{\prime} \mid S\right)+\sum_{S^{\prime} \in \mathcal{G}^{\prime}} P\left(S^{\prime} \mid S\right) \log M\left(S^{\prime}\right)
\end{gathered}
$$

for any fixed structure $S$. This further leads to

$$
\begin{aligned}
\sum_{S \in \mathcal{G}}\left(\begin{array}{l}
n \\
2
\end{array}\right) h(\epsilon) & P(S) \leq-\sum_{S \in \mathcal{G}} \sum_{S^{\prime} \in \mathcal{G}^{\prime}} P\left(S^{\prime} \mid S\right) \log P\left(S^{\prime} \mid S\right) P(S) \\
& +\sum_{S \in \mathcal{G}} \sum_{S^{\prime} \in \mathcal{G}^{\prime}} P\left(S^{\prime} \mid S\right) \log M\left(S^{\prime}\right) P(S) \\
= & -\sum_{S \in \mathcal{G}} \sum_{S^{\prime} \in \mathcal{G}^{\prime}} \log P\left(S^{\prime} \mid S\right) P\left(S, S^{\prime}\right) \\
& +\sum_{S^{\prime} \in \mathcal{G}^{\prime}} \log M\left(S^{\prime}\right) \sum_{S \in \mathcal{G}} P\left(S, S^{\prime}\right) \\
= & H\left(S^{\prime} \mid S\right)+\sum_{S^{\prime} \in \mathcal{G}^{\prime}} \log M\left(S^{\prime}\right) P\left(S^{\prime}\right) .
\end{aligned}
$$

In conclusion

$$
\left(\begin{array}{l}
n \\
2
\end{array}\right) h(\epsilon) \leq H\left(S^{\prime} \mid S\right)+\sum_{S^{\prime} \in \mathcal{G}^{\prime}} \log M\left(S^{\prime}\right) P\left(S^{\prime}\right) .
$$

Since $M\left(S^{\prime}\right)=\frac{n !}{\operatorname{Aut}\left(S^{\prime}\right)}$, from (6) we obtain

$$
\left(\begin{array}{l}
n \\
2
\end{array}\right) h(\epsilon) \leq H\left(S^{\prime} \mid S\right)+\log n !-\sum_{S^{\prime} \in \mathcal{G}^{\prime}} \log \left|\operatorname{Aut}\left(S^{\prime}\right)\right| P\left(S^{\prime}\right)
$$

leading to

$H\left(S^{\prime} \mid S\right) \geq-\log n !+\sum_{S^{\prime} \in \mathcal{G}^{\prime}} \log \left|\operatorname{Aut}\left(S^{\prime}\right)\right| P\left(S^{\prime}\right)+\left(\begin{array}{l}n \\ 2\end{array}\right) h(\epsilon)$.

This completes the proof of (iii).

\section{Proof of Theorem 1}

We are now ready to prove our main result, namely Theorem 1. We first establish an upper bound, and then derive the corresponding lower bound.

Theorem 2: The capacity of structural Binary Symmetric Channel $\operatorname{BSC}(\epsilon)$ satisfies

$$
C \leq 1-h(\epsilon) .
$$

Proof: Our goal is to estimate the mutual information $I\left(S ; S^{\prime}\right)=H\left(S^{\prime}\right)-H\left(S^{\prime} \mid S\right)$. We start with calculating $H\left(S^{\prime}\right)$ of $S^{\prime} \in \mathcal{G}^{\prime}(n, p * \epsilon)$. Observe that

$$
P\left(S^{\prime}\right)=\sum_{G^{\prime} \cong S^{\prime}, G^{\prime} \in \mathcal{G}} P\left(G^{\prime}\right)=M\left(S^{\prime}\right) P\left(G^{\prime}\right)
$$


where $M\left(S^{\prime}\right)=n ! /\left|\operatorname{Aut}\left(S^{\prime}\right)\right|$ is the number of different labeled graphs that have the same structure as $S^{\prime}$. Therefore, the structural entropy $H\left(S^{\prime}\right)$ of the random graph $\mathcal{G}^{\prime}(n, p * \epsilon)$ can be expressed as

$$
\begin{gathered}
H\left(S^{\prime}\right)=\mathbb{E}\left[-\log P\left(S^{\prime}\right)\right]=-\sum_{S^{\prime} \in \mathcal{G}^{\prime}} P\left(S^{\prime}\right) \log P\left(S^{\prime}\right) \\
=-\sum_{S^{\prime} \in \mathcal{G}^{\prime}} P\left(S^{\prime}\right) \log M\left(S^{\prime}\right) P\left(G^{\prime}\right) \\
=\sum_{S^{\prime} \in \mathcal{G}^{\prime}} P\left(S^{\prime}\right) \log \left|\operatorname{Aut}\left(S^{\prime}\right)\right|-\sum_{S^{\prime} \in \mathcal{G}^{\prime}} P\left(S^{\prime}\right) \log n ! \\
\quad-\sum_{S^{\prime} \in \mathcal{G}^{\prime}} P\left(S^{\prime}\right) \log P\left(G^{\prime}\right)=-\log n !+ \\
+\sum_{S^{\prime} \in \mathcal{G}^{\prime}} P\left(S^{\prime}\right) \log \left|\operatorname{Aut}\left(S^{\prime}\right)\right|-\sum_{G^{\prime} \in \mathcal{G}^{\prime}} P\left(G^{\prime}\right) \log P\left(G^{\prime}\right) \\
=-\log n !+\sum_{S^{\prime} \in \mathcal{G}^{\prime}} P\left(S^{\prime}\right) \log \left|\operatorname{Aut}\left(S^{\prime}\right)\right|+H\left(G^{\prime}\right) .
\end{gathered}
$$

Note that

$$
H\left(G^{\prime}\right)=-m E\left[\log P\left(X_{1}\right)\right]=\left(\begin{array}{l}
n \\
2
\end{array}\right) h(p * \epsilon)
$$

where $m=\left(\begin{array}{l}n \\ 2\end{array}\right)$ edges. Thus

$H\left(S^{\prime}\right)=-\log n !+\sum_{S^{\prime} \in \mathcal{G}^{\prime}} P\left(S^{\prime}\right) \log \left|\operatorname{Aut}\left(S^{\prime}\right)\right|+\left(\begin{array}{l}n \\ 2\end{array}\right) h(p * \epsilon)$.

On the other hand, from Lemma 2 (iii)

$H\left(S^{\prime} \mid S\right) \geq-\log n !+\sum_{S^{\prime} \in \mathcal{G}^{\prime}} \log \left|\operatorname{Aut}\left(S^{\prime}\right)\right| P\left(S^{\prime}\right)+\left(\begin{array}{l}n \\ 2\end{array}\right) h(\epsilon)$.

Therefore, combining it with the fact that $I\left(S ; S^{\prime}\right)=$ $H\left(S^{\prime}\right)-H\left(S^{\prime} \mid S\right)$, we have

$$
I\left(S ; S^{\prime}\right) \leq\left(\begin{array}{l}
n \\
2
\end{array}\right)[h(p * \epsilon)-h(\epsilon)] .
$$

In summary

$$
C=\lim _{n \rightarrow \infty} \frac{1}{\left(\begin{array}{c}
n \\
2
\end{array}\right)} \max _{0 \leq p \leq 1} I\left(S ; S^{\prime}\right) \leq 1-h(\epsilon)
$$

which is the desired upper bound.

Now we establish the corresponding lower bound.

Theorem 3: The capacity of structural Binary Symmetric Channel $\operatorname{BSC}(\epsilon)$ satisfies

$$
C \geq 1-h(\epsilon)
$$

Proof: Observe that the capacity of the labeled graph transmission over a $\operatorname{BSC}(\epsilon)$ channel is

$$
C_{L}=\lim _{n \rightarrow \infty} \max _{0 \leq p \leq 1} \frac{1}{\left(\begin{array}{c}
n \\
2
\end{array}\right)} I\left(G ; G^{\prime}\right) .
$$

To compute the mutual information $I\left(G ; G^{\prime}\right)=H\left(G^{\prime}\right)-$ $H\left(G^{\prime} \mid G\right)$, we first observe that by Lemma 1 (iv)

$$
H\left(G^{\prime} \mid G\right)=\left(\begin{array}{l}
n \\
2
\end{array}\right) h(\epsilon) .
$$

Moreover, when $G$ is an Erdôs-Rényi graph $\mathcal{G}(n, p)$, then $G^{\prime}$ is an Erdős-Rényi graph $\mathcal{G}(n, p * \epsilon)$. Therefore, by (11) we have

$$
H\left(G^{\prime}\right)=\left(\begin{array}{l}
n \\
2
\end{array}\right) h(p * \epsilon) .
$$

Thus

$$
I\left(G ; G^{\prime}\right)=\left(\begin{array}{l}
n \\
2
\end{array}\right)[h(p * \epsilon)-h(\epsilon)] .
$$

Clearly,

$$
\lim _{n \rightarrow \infty} \max _{0 \leq p \leq 1} \frac{1}{\left(\begin{array}{c}
n \\
2
\end{array}\right)} I\left(G ; G^{\prime}\right)=\max _{0 \leq p \leq 1} h(p * \epsilon)-h(\epsilon)
$$

hence $C_{L}=1-h(\epsilon)$. This means that we can transmit labeled graphs up to the rate $C_{L}=1-h(\epsilon)$ per channel transmission with the average error probability

$$
P_{1}^{(n)}(e)=P\left(G^{\prime} \neq G\right) \rightarrow 0 .
$$

Now, re-consider the transmission model in Figure 1, i.e.,

$$
S \rightarrow G \rightarrow G^{\prime} \rightarrow S^{\prime}
$$

In this model (i.e., SBSC), it is clear that we can transmit structures at the same transmission rates as labeled graph case by performing the process (11) since the average error probability in this case satisfies

$$
0 \leq P_{2}^{(n)}(e)=P\left(S^{\prime} \neq S\right) \leq P\left(G^{\prime} \neq G\right)=P_{1}^{(n)}(e) \rightarrow 0
$$

since $S^{\prime} \neq S$ implies $G^{\prime} \neq G$. Hence, $P_{2}^{(n)}(e) \rightarrow 0$. Consequently, $C \geq C_{L}=1-h(\epsilon)$.

\section{ACKNOWLEDGMENT}

This work was supported in part by the NSF Science and Technology Center for Science of Information Grant CCF0939370, NSF Grant CCF-0830140, AFOSR Grant FA865511-1-3076, and NSA Grant H98230-11-1-0141.

\section{REFERENCES}

[1] B. Bollobas, Random Graphs, Cambridge University Press, 2001.

[2] F. Brooks. Three great challenges for half-century-old computer science. J. the ACM, 50:25-26, 2003.

[3] Y. Choi and W. Szpankowski, Compression of Graphical Structures: Fundamental Limits, Algorithms, and Experiments," IEEE Trans. Inf. Theory, vol. 58, no. 2, pp. 620-638, 2012.

[4] T. Cover and J. Thomas, Elements of Information Theory, 2nd ed.,New Jersey: John Wiley \& Sons, Inc., 2006.

[5] P. Erdős and A. Rényi, Asymmetric graphs, Acta Math. Acad. Sci. Hungar, vol. 14, pp. 295-315, 1963.

[6] J. Kieffer, E-H. Yang, and W. Szpankowski, Structural Complexity of Random Binary Trees 2009 International Symposium on Information Theory, 635-639, Seoul, 2009.

[7] M. Naor, Succinct representation of general unlabeled graphs, Discrete Applied Mathematics, 28(3), 303-307, 1990.

[8] P. Nurse. Life, logic, and information. Nature, 454:424-426, 2008.

[9] C. E. Shannon, A Mathematical Theory of Communications, Bell Syst. Tech. , J., 27:379-423, 623-656, 1948.

[10] W. Szpankowski, Algorithms, Combinatorics, Information, and Beyond, IEEE Information Theory Society Newsletter, 62, 5-20, 2012.

[11] Gy. Turan, On the succinct representation of graphs, Discrete Applied Mathematics, 8(3), 289-294, 1984. 